Computing and Informatics, Vol. 40, 2021, 729-733, doi: 10.31577/cai_2021_4_729

\title{
PREFACE TO THE SPECIAL ISSUE ON SOFTWARE ENGINEERING
}

\author{
Marian Bubak, Maciej Malawski \\ Sano Centre for Computational Medicine \\ Kraków, Poland \\ E) \\ Institute of Computer Science, AGH University of Science and Technology \\ Kraków, Poland \\ e-mail: $\{$ m.bubak, m.malawski\}@sanoscience.org
}

\author{
Renata G. SŁOtA \\ Institute of Computer Science, AGH University of Science and Technology \\ Kraków, Poland \\ e-mail: renata.slota@agh.edu.pl
}

This special issue is composed of carefully selected papers presented at the XXII KKIO Software Engineering Conference organized in Krakow on 21-22 September 2021 (https://kkio.pti.org.pl/2021/). KKIO is a prime software engineering international conference organized annually by the Polish Information Processing Society (https://portal.pti.org.pl) in a close collaboration with different Polish university computer science institutes. This edition of KKIO was organized by the Malopolska Branch of the Polish Information Processing Society and Institute of Computer Science AGH University of Science and Technology, Krakow, Poland.

The aim of KKIO is to stimulate research and to promote cooperation between science, industry, and governmental bodies. It is a platform to make contacts, initiate cooperation between researchers and engineers, and draft national and international projects. This conference allows managerial staff from the industry, private organizations, and government bodies to widen their knowledge about software engineering to learn the latest achievements in software engineering research and praxis.

The papers in this issue represent seven most successful topics of the Conference.

Topic Software Engineering Processes, Practices, Methods, and Tools has gathered contributions related to new tools, processes, practices, methods, and tools 
supporting software life cycles as well as evaluation or improvement of the new or existing processes, practices, methods, and tools. This topic is represented by the paper

- Krzysztof Marek, Michał Śmiałek, Kamil Rybiński, Radosław Roszczyk and Marek Wdowiak: BalticLSC: Low-Code Software Development Platform for Large Scale Computations [1].

Topic Automating Software Engineering addressed development of contemporary software systems as a complex enterprise which requires automated approaches to be done in an economic and timely manner. This is not possible without techniques for constructing and transforming software artifacts and supporting software processes with tools. Two different approaches are described in the papers

- Maciej Penar and Wiktor Zychla: Object-Oriented Build Automation - A Case Study [2],

- Bogumila Hnatkowska and Mateusz Cebinka: Activity Diagram Generation Based on Use-Case Textual Specification [3].

Topic Software Engineering for Particle Physics was proposed for the first time at the KKIO Software Engineering Conference, and it resulted in two interesting keynote lectures and a number of valuable presentations. This field is characterized by a set of information technology challenges related to exabyte data volumes and millions of computing intensive simulation jobs both distributed around the World and therefore elaboration of optimised software solutions is necessary. We have decided to include in this issue three representative papers:

- Jakub Czajka, Jacek Otwinowski and Jacek Kitowski: Non-Intrusive Data Inspection for Message-Based Systems [4,

- Michał Mazurek, Gloria Corti and Dominik Muller: New Simulation Software Technologies at the LHCb Experiment at CERN [5],

- Aleksandra Poreba: The Trigger Performance Monitoring and Rate Predictions Preparation for Run 3 at Atlas Experiment [6].

Topic Software Engineering for Computational Medicine addressed development of software tools supporting prevention, diagnosis, and treatment of diseases, ultimately resulting in clinical decision support systems. It is the subject of the paper

- Jakub Adamczyk and Filip Malawski: Comparison of Manual and Automated Feature Engineering for Daily Activity Classification in Mental Disorder Diagnosis [7].

Topic Software Engineering for Network Services and Clouds gathered presentations related to methods of development for network-centric application, quality assurance and evaluation, architectures of cloud- and network-based applications and services in network environment. These challenges and solutions are described in the paper 
- Hanna Grodzicka, Michal Kedziora and Lech Madeyski: Cloud Solutions for Private Permissionless Blockchain Deployment [8].

Topic Software Engineering for Quantum Computing was included into the Conference to provide a forum for software developers, computer scientists and physicists to understand and discuss research on current problems in quantum software development. These aspects of quantum computing are presented in the paper

- Julia Plewa, Joanna Sieńko and Katarzyna Rycerz: Variational Algorithms for Workflow Scheduling Problem in Gate-Based Quantum Devices [9].

Finally, the paper

- Jacek Dajda et al.: Current Trends in Software Engineering Bachelor Theses [10]

describes the most significant results of the Competition of students' engineering projects which was organized to encourage graduates of engineering first-cycle degree studies to develop their professional aspirations in the context of software engineering and to enable the exchange of knowledge and experience of different academic communities.

\section{Acknowledgements}

We are very grateful to the members of the KKIO 2021 Program and Organization Committees for their contribution to elaboration of the Conference program and for reviewing the submitted papers. We thank the Malopolska Branch of the Polish Information Processing and Institute of Computer Science AGH for their generous support. The authors are grateful for support from the subvention of Polish Ministry of Education and Science assigned to AGH University of Science and Technology (Faculty of Computer Science, Electronics and Telecommunications).

We acknowledge the great work of Jarosław Koźlak in collecting and assessing submitted papers. Unfortunately, during the conference, he suddenly passed away. He will be missed very much as a colleague, teacher and scientist as well as a kind and helpful human being.

\section{REFERENCES}

[1] Marek, K.-ŚmiaŁek, M.-Rybiński, K.-Roszczyk, R.-Wdowiak, M.: BalticLSC: Low-Code Software Development Platform for Large Scale Computations. Computing and Informatics. Vol. 40, 2021, No. 4, pp. 734-753, doi: 10.31577/cai_2021_4_734.

[2] Penar, M.-Zychla, W.: Object-Oriented Build Automation - A Case Study. Computing and Informatics. Vol. 40, 2021, No. 4, pp. 754-771, doi: 10.31577/cai_2021_4_754 
[3] Hnatkowska, B.-Cebinka, M.: Activity Diagram Generation Based on Use-Case Textual Specification. Computing and Informatics. Vol. 40, 2021, No. 4, pp. 772-795, doi: 10.31577/cai_2021_4_772.

[4] Czajka, J.-Otwinowski, J.-Kitowski, J.: Non-Intrusive Data Inspection for Message-Based Systems. Computing and Informatics. Vol. 40, 2021, No. 4, pp. 796-814, doi: $10.31577 /$ cai_2021_4_796.

[5] Mazurek, M.-Corti, G.-Müller, D.: New Simulation Software Technologies at the LHCb Experiment at CERN. Computing and Informatics. Vol. 40, 2021, No. 4, pp. 815-832, doi: 10.31577/cai_2021_4_815.

[6] Poreba, A.: The Trigger Performance Monitoring and Rate Predictions Preparation for Run 3 at Atlas Experiment. Computing and Informatics. Vol. 40, 2021, No. 4, pp. 833-849, doi: 10.31577/cai_2021_4_833.

[7] Adamczyk, J.-Malawski, F.: Comparison of Manual and Automated Feature Engineering for Daily Activity Classification in Mental Disorder Diagnosis. Computing and Informatics. Vol. 40, 2021, No. 4, pp. 850-879, doi: 10.31577/cai_2021_4_850

[8] Grodzicka, H.-Kedziora, M.-Madeyski, L.: Cloud Solutions for Private Permissionless Blockchain Deployment. Computing and Informatics. Vol. 40, 2021, No. 4, pp. 880-896, doi: $10.31577 /$ cai_2021_4_880.

[9] Plewa, J.-Sieńko, J.-Rycerz, K.: Variational Algorithms for Workflow Scheduling Problem in Gate-Based Quantum Devices. Computing and Informatics. Vol. 40, 2021, No. 4, pp. 897-929, doi: 10.31577/cai_2021_4_897.

[10] Dajda, J.--Idzik, M.-SrokA, J.-Sikora, M. et al.: Current Trends in Software Engineering Bachelor Theses. Computing and Informatics. Vol. 40, 2021, No. 4, pp. 930-956, doi: 10.31577/cai_2021_4_930. 


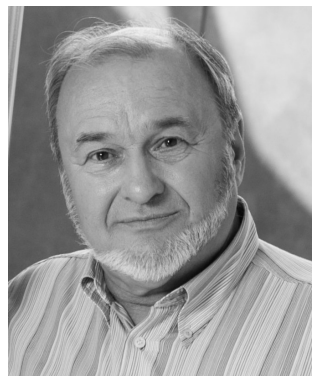

Marian BubAK is Scientific Affairs Director of the Sano Centre for Computational Medicine, he also leads the Laboratory of Information Methods in Medicine at the ACC Cyfronet AGH, a staff member of the Institute of Computer Science AGH, and Professor of Distributed System Engineering (Emeritus) at the Informatics Institute University of Amsterdam. He obtained his M.Sc. in technical physics and Ph.D. in computer science from the AGH University of Science and Technology, Krakow, Poland. His research interests include parallel and distributed computing, problem solving environments, and quantum computing. He authored about 250 papers in these areas and co-edited a number of books. He served key roles in series of EU-funded projects, as a member of editorial boards of FGCS, Bio-Algorithms and Med-Systems, and Computer Science Journal as well as chairman/organizer of international conferences (including ICCS in 2004 and 2008). Since 2017 he is the chairman of the Malopolska Branch of the Polish information Processing Society.

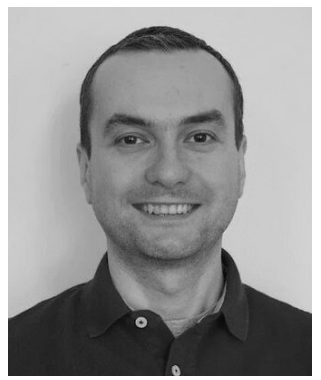

Maciej MalawSKI is Leader of Extreme Scale Data and Computing at Sano and Associate Professor at the Institute of Computer Science AGH. He is co-author of over 50 international publications including journal and conference papers, and book chapters. Member of technical program committees of premier conferences on scientific, parallel and distributed computing (SC, ICCS, CCGrid, UCC, IPDPS), general co-chair of EuroPar 2020. His scientific interests include parallel computing, cloud and serverless technologies, distributed systems, workflows, resource management and scientific applications, with particular focus on high energy physics (CERN) and biomedical research (Sano).

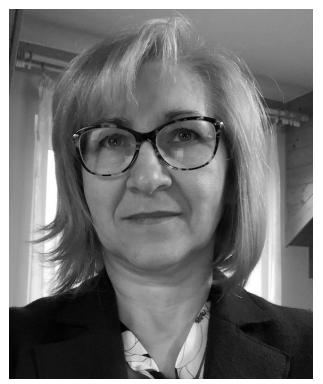

Renata G. SŁota is Associate Professor at the Institute of Computer Science, AGH University of Science and Technology (AGH-UST). She was (2016-2020) Deputy Dean at the Faculty of Computer Science, Electronics and Telecommunications AGH-UST and now is Coordinator for the Quality of Education in the scientific discipline of Information and Communication Technology at AGH UST. Co-author of over 150 international publications including journal and conference papers, and book chapters. She has participated in many EU-FP5/FP6/ FP7/H2020 and national research projects. Her scientific interests relate to the area of data storage systems, data sharing and management for large grid and cloud infrastructures, computing environments and platforms facilitating access to HPC infrastructures. 